\title{
Percepción del riesgo ambiental en mega proyectos acuícolas. El caso de la comuna de Cobquecura en Chile ${ }^{1}$
}

\author{
Environmental risk perception in aquaculture \\ mega-projects. The case of Cobquecura in Chile
}

\author{
Paulina Alarcón², Álvaro Carrasco ${ }^{3}$, César Salazar $^{4}$ [0, Marcela Jaime ${ }^{5}$ (1) \\ y Cristina Martin 6
}

\begin{abstract}
RESUMEN
La acuicultura se ha convertido en una actividad clave para el desarrollo costero. Pese a sus efectos positivos sobre el empleo, es cuestionada por los efectos que genera en el entorno. Este trabajo analiza las motivaciones de una comunidad rural en Chile para rechazar la instalación de una empresa acuícola. Los resultados revelan que riesgos de impactos ambientales, cambios en la forma de vida y organización del trabajo, disminución del turismo, y demanda de trabajo especializada explican este rechazo. Experiencias pasadas de la industria en el sur de Chile, y un insuficiente diálogo entre la comunidad y la empresa refuerzan esta percepción.
\end{abstract}

Palabras clave: acuicultura, conflictos sociales, comunidades rurales, Chile.

\section{ABSTRACT}

Aquaculture has become a key activity for coastal development. Despite of positive impacts on employment, it is questioned by its potential negative effects. This paper analyses the motivations of a rural community in Chile to reject the installation of an aquaculture firm. The results reveal that potential environmental impacts, changes in the way of life and organization of work, tourism reduction, and the demand of specialized labor explain this rejection. Past experiences of the industry in southern Chile, and insufficient dialogue between the community and the company reinforce this perception.

Keywords: aquaculture, social conflicts, rural communities, Chile.

Se agradece el apoyo financiero a los proyectos FONDAP N015110027 y CONICYT PIA/BASAL FBO002

Departamento de Gestión Empresarial, Facultad de Ciencias Empresariales, Universidad del Bío-Bío. Correo electrónico: paulina.alarcon1401@egresados.ubiobio.cl

Departamento de Gestión Empresarial, Facultad de Ciencias Empresariales, Universidad del Bío-Bío. Correo electrónico: alvaro.carrasco1401@egresados.ubiobio.cl

Departamento de Gestión Empresarial, Facultad de Ciencias Empresariales e investigador, Centro Interdisciplinario para la Investigación Acuícola (INCAR), Centro de Estudios de Ñuble, Universidad del Bío-Bío y Environment for Development EfD-NENRE Correo electrónico: csalazar@ubiobio.cl

Escuela de Administración y Negocios, Universidad de Concepción e investigadora Environment for Development EfD-NENRE y Centro de Ecología Aplicada y Sustentabilidad (CAPES), Correo electrónico mjaime@udec.cl

Observatorio Laboral Ñuble, Universidad del Bío-Bío. E-mail: crmartin@ubiobio.cl. 
La acuicultura es una actividad técnica y controlada para el cultivo de peces, moluscos y algas en zonas costeras y aguas interiores. En la actualidad, este es el sector de más rápido crecimiento respecto a la producción de alimentos en el mundo (FAO, 2016). Chile, con sus 4.300 kilómetros de territorio colindantes al océano pacifico, disponibilidad de agua y perfectas condiciones sanitarias, ha aprovechado este potencial para situarse como el segundo mayor productor mundial de salmón (Buschmann, 2001). Esta actividad productiva ha mostrado efectos económicos positivos que se han traducido en creación de empleos para mujeres y jóvenes (Amtmann \& Blanco, 2017). También la evidencia muestra que la instalación de centros de cultivos ha contribuido a reducir la pobreza en Chile en las localidades rurales donde han operado (Ceballos et al., 2018).

Por otro lado, la industria del salmón es una actividad que compite por el espacio marino con otros usos alternativos tanto productivos como no productivos (Chávez et al., 2019). Esta situación genera un conflicto con las comunidades aledañas a las localidades donde opera. La tensión se produce principalmente por el alto riesgo de degradación ambiental que genera la industria, y efectos socioeconómicos no deseados, geográficamente limitados a la población de las localidades donde opera (Barton \& Murray, 2009; ICES, 2011). Los efectos ambientales se materializan en externalidades negativas que surgen como consecuencia de la generación de residuos desde las fecas y alimento para los salmones (Buschmann et al., 2006; Soto \& Norambuena, 2004), uso indiscriminado de antibióticos y otros productos químicos con efectos nocivos no solo para el ambiente sino también para la salud de las personas (Buschmann \& Fortt, 2005; Miranda \& Rojas, 2007; Cabello et al., 2013; Millanao et al., 2011), y escapes de salmones con riesgos de depredación y mayor competencia por alimento con especies nativas (Niklitschek et al., 2013; Sepúlveda et al., 2013). Con respecto a los efectos socioeconómicos, se argumenta que la instalación de una industria transnacional y moderna podría tener un profundo impacto sobre la forma de vida de las comunidades, generando una disrupción cultural (Claude et al., 2000; Barrett et al., 2002).

El objetivo de este trabajo es explorar las motivaciones de una comunidad local para rechazar la posible instalación de una industria acuícola en su territorio. El caso de estudio, foco de esta investigación, se desarrolla en la comuna de Cobquecura de la Región de Nuble, Chile. Cobquecura, con un $45 \%$ de sus habitantes bajo la línea de la pobreza, es una de las comunas más rezagadas de la Región de Ñuble y el país (FLACSO, 2018). Esta condición de rezago alienta la necesidad de promover la instalación de empresas en la zona. En este contexto, en el año 2016, se presentó para la comuna uno de los proyectos acuícolas más ambiciosos del país, el cual busca el desarrollo del cultivo de salmones, cojinova del norte, choritos y huiro, a través de la instalación de 11 centros de cultivos (Villalobos, 2016). El proyecto, que a la fecha de esta investigación se encontraba en etapa de evaluación de impacto ambiental, ha generado un profundo rechazo de la comunidad local. Uno de los argumentos principales que fundamenta este rechazo en el potencial riesgo de daño ambiental del patrimonio natural de la comuna como consecuencia de la instalación de la industria acuícola en la zona. Uno de estos patrimonios corresponde a la existencia de una de las loberías reproductivas más importantes del cono sur, protegida por ley, y declarado santuario de la naturaleza en 1992. Este santuario tiene un gran valor para las comunidades locales. Además, en la comuna, se encuentran numerosos humedales y estuarios con relevancia desde el punto vista ambiental, siendo un hábitat importante para una gran cantidad de aves migratorias.

Esta investigación se enmarca en el concepto de sociedad del riesgo, en el sentido que la posible instalación de esta industria en la zona es un suceso con cierta probabilidad de ocurrencia. Cuando hacemos uso de la noción de riesgo para observar un fenómeno, éste puede ser entendido 
como algo que no solo ha sido generado (o puede generarse) por la acción humana, sino que también puede evitarse mediante la misma. Sin embargo, lo que lo hace el análisis de este fenómeno aún más interesante, es la existencia de diversas corrientes de opinión que consideran que el que éste $u$ otro suceso se verifique o no depende de nuestras acciones en el presente. Por lo tanto, catalogar a algo como riesgoso involucra transformar la realidad a tal punto que nunca podremos saber si, efectivamente, un determinado curso de acción nos hubiera llevado a la catástrofe (Galindo, 2015). Lo que las sociedades tradicionales atribuían a la fortuna, voluntad divina o destino; la sociedad moderna lo atribuye al riesgo, entendiendo por éste, la posibilidad contenida en cierto curso de acción social, de generar efectos adversos (Cernea, 2001). El riesgo aparece como un constructo social histórico en la transición de la baja edad media a la edad moderna temprana. Este constructo se basa en la determinación de lo que la sociedad considera en cada momento como normal y seguro (Giddens et al., 1996). El concepto de riesgo delimita, por tanto, un peculiar estado intermedio entre seguridad y destrucción, donde la percepción de riesgos amenazantes determina pensamiento y acción (Beck, 2000). En ese sentido, Beck (2000) analiza la transformación estructural sufrida por los conflictos sociales en las sociedades industrializadas, y afirma que en ella los conflictos sociales ya no se relacionan con el tema de la distribución de la riqueza, sino con la distribución de los riesgos. Por consiguiente, la "sociedad de riesgo" es un conjunto particular de condiciones sociales, económicas, políticas y culturales que se caracterizan por la lógica cada vez más generalizada de la incertidumbre que conlleva a la transformación de las estructuras, las instituciones y las relaciones sociales existentes, hacia una incorporación de mayor complejidad, contingencia y fragmentación (Beck and Rey, 2002). Es así como, mientras la sociedad del riesgo conlleva un sentido de dominación institucional (de ahí la "sociedad"), la cultura del riesgo abarca todos los tipos de formas residuales y marginales de las prácticas de creación de sentido (Adam et al., 2000). La posible instalación de megaproyectos en espacios rurales y zonas naturales emerge como uno de estos riesgos. La discusión frente a estos megaproyectos es motivada por las consecuencias y los costos-beneficios que suponen la construcción de estos proyectos. Los costos se relacionan con cambios significativos en la vida de las comunidades, en lo que se refiere a presión de tierras, conflictos de propiedad, migración, empobrecimiento, exclusión social y pérdida de identidad (González, 2009). Lo paradójico de hablar de conflictos producidos por mega proyectos en las comunidades radica en que se producen por la ausencia o presencia de estos. Debido a que, algunos sectores los visualizan como posibilidades de desarrollo económico, mientras que otros los asocian con amenazas irreparables al medio ambiente (Rincón, 2016).

La mayoría de los megaproyectos que generan conflictos sociales han estado ligados a la explotación de los recursos naturales, principalmente proyectos mineros y energéticos que se desarrollan con tecnologías de extracción, poniendo en riesgo el patrimonio histórico cultural y ambiental de las comunidades aledañas (Vargas, 2009). Dicha puesta en valor de la naturaleza deviene un malestar frente al modelo de desarrollo basado en la extracción de recursos naturales (Jaimovich et al., 2014). De esta manera, los efectos de la instalación de megaproyectos también pueden ser analizados desde la perspectiva de los riesgos ambientales. Los riesgos ambientales no solo están determinados por factores económicos, sino también por factores tecnológicos, ambientales y de comportamiento (Ezzati et al., 2005). Estos riegos son inherentes a proyectos de todo tipo, incluso en aquellos diseñados como soluciones inclusivas a problemáticas relevantes para una localidad. Yenneti et al. (2016) evidencian, por ejemplo, que los costos de la instalación de un gran parque de energía solar en India podrían superar sus beneficios, ya que su implementación aumentaría la precariedad de comunidades vulnerables, quienes podrían no solo perder 
su fuente de sustento, sino también ser desplazados de su lugar de residencia. Si bien existe un consenso de que la mayoría de los megaproyectos traen consigo el desplazamiento de las comunidades (Yenneti et al., 2016), algunos proyectos en particular podrían afectar principalmente la dinámica social de las comunidades, tal y como sucede en el caso del desarrollo del turismo (Allen et al., 1988) o la dinámica ambiental y de los ecosistemas provistos en las localidades de residencia, lo cual es especialmente relevante en el caso de los proyectos acuícolas (Olsen \& Osmundsen, 2019; Couch \& Kroll-Smith, 1994). La literatura evidencia también la importancia de la acción colectiva, las redes sociales y el acceso a información para contrarrestar y/o mitigar los efectos nocivos de los riesgos ambientales (Ireland \& Thomalia, 2011; Chilvers, 2009; Failing et al., 2007; Gouldson, 2004; Wakefield et al., 2001). Un análisis de estos determinantes en forma conjunta permite conocer, en mayor detalle, las percepciones del nivel de exposición de las comunidades, y contribuir a una evaluación más objetiva en torno a la implementación de un proyecto en particular.

Entre los numerosos conflictos ambientales y megaproyectos en Chile, destaca el proyecto hidroeléctrico Ralco, el cual pone en riesgo un ecosistema de gran valor y diversidad junto con la sobrevivencia cultural y los derechos de los pehuenches, pueblo originario de Chile (Rojas et al., 2003). El sector forestal es otro de los sectores que ha enfrentado conflictos medioambientales en el país. Un ejemplo, es el caso de la planta Valdivia de celulosa Arauco, CELCO, que desde el momento de su instalación generó cuestionamientos a nivel regional y nacional por su localización cercana a un santuario de la Naturaleza Carlos Anwandter (Cuenca, 2005). La industria acuícola en Chile también ha sido objeto de conflictos sociales, al ofrecer desarrollo y progreso económico en pos de un desarrollo social, por un lado, y por otro, transformar distintos espacios socio-territoriales, en relación con su organización social, estructura familiar, mercado productivo, dinámica del trabajo, tradiciones culturales, entre otras (; Barrett et al., 2002; Amtmann \& Blanco, 2017; Ceballos et al., 2018). Alfaro \& Peña-Cortés (2012) identifican la existencia de zonas de conflicto por el uso de las aguas interiores del área pre-andina de la Región de La Araucanía en Chile para actividades de turismo y salmonicultura.

Es así como, la comuna de Cobquecura se ve inmersa en una sociedad de riesgo, ya que se encuentra en un estado de incertidumbre que provoca un cambio principalmente en el comportamiento de las instituciones y relaciones sociales. Este cambio en el comportamiento es motivado por la percepción que tiene la comunidad frente a la amenaza de seguridad o destrucción de sus costas, en el marco de los nuevos desafíos que trae consigo el desarrollo económico e industrial.

\section{Área de estudio}

El contexto en el que se realiza esta investigación es la gran incertidumbre que la comuna de Cobquecura (región de Ñuble en Chile) ha experimentado frente a la inminente instalación de una empresa acuícola. Esta comuna posee una población de 5.012 habitantes. Es un territorio de tradición e identidad ligada históricamente a lo rural, donde un $75 \%$ de los habitantes reside principalmente en zonas rurales, distribuyéndose en diversos poblados. Esta comuna posee características particulares, que la convierten en un lugar en donde lo rural y urbano converge, favoreciendo las formas de intercambio económico, social, cultural, entre otras. Existe una fuerte interacción entre la pesca y la agricultura, que se lleva a cabo con el máximo respeto a las espe- 
cies marinas autóctonas, como el lobo marino, que es la especie protegida símbolo de la zona. En los meses de verano, alberga una fuerte cantidad de turistas que llegan principalmente para disfrutar de la naturaleza autóctona y practicar deportes acuáticos como surf y bodyboard (Municipalidad de Cobquecura, 2018). La actividad turística se sustenta principalmente en su litoral, que posee una biodiversidad única, y por cuyas costas transitan constantemente diferentes mamíferos marinos como ballenas y delfines, además de ser muy productivas en bancos de mariscos y diferentes tipos de peces. En efecto, la creciente llegada de turistas en los últimos años, hace que esta comuna establezca el sector turismo (hoteles, restoranes, artesanía) como una fuente de ingresos importante para su economía, así como el sector silvo-agropecuario, con una economía tradicional y trabajos del sistema público dependientes de la municipalidad.

\section{Materiales y Métodos}

\section{Tipo de estudio}

El método que se utiliza en esta investigación es el estudio de caso. Este método se elige porque permite analizar un fenómeno en profundidad, y plantearse una descripción de la situación real, asociado a un fenómeno contemporáneo y que ha sido socializado por el grupo al cual pertenece. Se conceptualiza, por tanto, como una investigación sistemática e intensiva, típicamente conducida bajo condiciones naturales, relacionándolos con antecedentes, situaciones comunes, características del ambiente y sus posibles interacciones (Urra et al., 2014). De acuerdo con Stake (1999), el cometido real del estudio de casos es la particularización, no la generalización, en donde se toma un caso particular para conocerlo en profundidad, a diferencia del enfoque de generalización, donde lo importante son las similitudes o diferencias con otros estudios.

Figura $\mathrm{N}^{0} 1$.

Localización de la zona de estudio

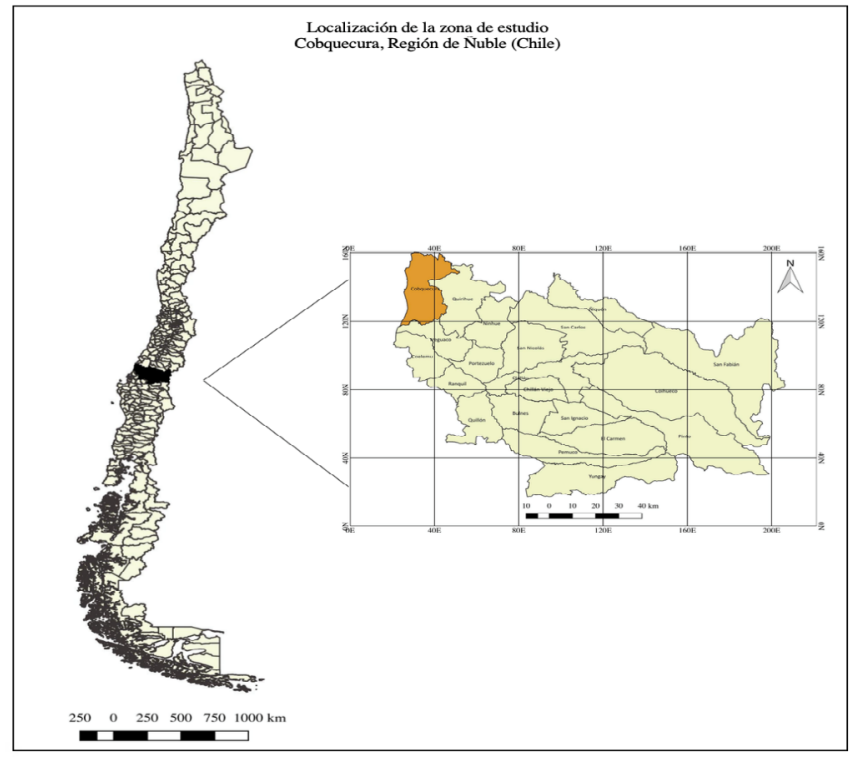

Fuente: Elaboración propia. 


\section{Técnicas de levantamiento de información}

Las técnicas que se utilizan en este estudio de caso son de carácter cualitativo, con un enfoque fenomenológico. El alcance del estudio es descriptivo, puesto que relata las impresiones de los distintos habitantes de la comuna de Cobquecura, describiendo, comprendiendo e interpretando situaciones y percepciones entregadas por estos. De carácter fenomenológico, ya que los hechos fueron considerados como un fenómeno en particular, con características específicas que los diferencian de otros hechos, lo que permite conocer la realidad "in situ" y compartir experiencias en el ambiente estudiado, logrando captar la percepción de los actores y obtener una visión general y argumentada de sus motivaciones para rechazar una posible instalación de una industria acuícola (Pellicer et al., 2013).

En lo que respecta al trabajo de campo, se opta por un muestreo no probalístico, con la ayuda de informantes claves de la comunidad, utilizando el método de la bola de nieve (Goodman, 1961). Este método permite acceder a un contacto directo con diferentes grupos de actores. Las técnicas utilizadas para la recolección de información son: (1) entrevista a actores claves de la comuna de Cobquecura, y (2) grupos focales. Ambos enfoques permiten analizar en mayor profundidad las motivaciones de los habitantes de la zona de estudio. Por una parte, la entrevista a actores relevantes permitió rescatar con mayor profundidad las motivaciones de carácter individual de las personas, con el fin de obtener un mayor grado de información e influencia sobre algunos grupos u organizaciones. Los grupos focales, a su vez, permitieron obtener representaciones de carácter colectivo de los relatos, donde la tarea grupal consistió en el consenso hacia la postura frente a la instalación de la industria acuícola. La recogida de información se realizó durante los meses de noviembre y diciembre del año 2018. La muestra de entrevistados se compuso de siete actores claves, residentes de la comuna, y con participación activa en ámbitos tanto municipales, educacionales, como también, gastronómicos, hoteleros y agrícolas. Asimismo, se realizaron dos grupos focales, totalizando catorce personas, pertenecientes a diferentes organizaciones de la sociedad civil, junta de vecinos, comité de agua potable, clubes deportivos y sindicatos. El análisis se llevó a cabo a partir de la elaboración de una guía temática, que busca la profundización en los aspectos socioculturales, ambientales y económicos que percibe la comunidad al exponer su visión frente a su rechazo a la posible instalación de este megaproyecto acuícola en la comuna.

\section{Resultados}

\section{Motivaciones individuales a través de entrevistas a actores clave}

Con el objeto de poder tener una visión general de las motivaciones de los habitantes de Cobquecura ante la instalación de la industria, en primer lugar, se analizan las percepciones de un conjunto diverso de actores que ejercen influencia en los distintos ámbitos del quehacer de la comuna. Los resultados se organizan en función de las tres dimensiones de interés (i.e., ambiental, económica y socio-cultural).

En relación a la dimensión ambiental, una posible instalación de una industria acuícola en la comuna es percibida por parte de la comunidad como un riesgo innecesario que amenaza el ecosistema en general. 
"Me parece muy riesgoso, me parece que es una amenaza innecesaria puesto que ya está demasiado comprobado el daño que se produce con esta especie foránea, principalmente dañando lo que es medio ambiente pero también actuando como depredadores lo que obviamente como impacto va a generar un retroceso en lo que es desarrollo turístico de la comuna" (Fuente: encuestado de la zona).

En particular, los entrevistados manifiestan un rechazo a la industria motivados por el potencial impacto negativo que podía tener en el ecosistema de Cobquecura, haciendo énfasis en los efectos negativos que podría causar al santuario de la naturaleza, constituido por una de las loberías reproductivas más importantes del cono sur. Otro de los aspectos ambientales que se destaca y se tiene consciencia, se relaciona con el hecho de que las costas de Cobquecura presentan un tránsito constante de cardúmenes de diversas especies comerciales y no comerciales. Entre las no comerciales, se encuentran mamíferos amenazados como ballenas y delfines. Los entrevistados también dan cuenta de una postura contraria a la forma de producción de salmones en Chile, argumentando que existe un uso excesivo de antibióticos que se acumulan en el suelo marino con efectos negativos sobre la flora y fauna de los océanos.

Asimismo, los habitantes de la zona relacionan las experiencias de la industria del salmón instalada en otras regiones del país, en términos de desastres ambientales en los que esta actividad ha sido señalada como responsable (o dentro del grupo de agentes responsables), con lo que podría suceder en Cobquecura.

"Después vino esta otra crisis que le llamaron la marea roja, pero fue debido también a que murieron muchos salmones por falta de oxígeno, porque la naturaleza es sabia y creó sobre toda esta materia orgánica del fondo marino un alga que le invadió el oxígeno a los salmones, entonces hubo esta tremenda mortalidad y que ellos vertieron muy cerca de las costas... y eso fue lo que mato todos los mariscos." (Fuente: encuestado de la zona).

Desde una perspectiva económica, la instalación de la empresa acuícola también es rechazada por los actores locales, manifestando que la creación de empleos sería insuficiente y que no beneficiaría directamente a la comuna, ya que la industria requiere de mano de obra especializada que no se encuentra en la zona. Los habitantes tampoco manifiestan una actitud positiva a poder tomar estos empleos. Asimismo, se plantea que se produciría una reducción importante de fuentes de trabajo ligadas a la actividad turística y sus encadenamientos productivos con la pesca y agricultura, producto de sus potenciales efectos negativos sobre el ambiente.

"Claramente el turismo va a ser menoscabado y los pequeños empresarios y los pescadores y la gente que vende productos agrícolas se van a ver perjudicados, entonces no hay una congruencia entre un proyecto que dice que va a generar trabajo que van hacer alrededor de sesenta personas más menos lo que se sabe ya, a donde vive una comuna entera del turismo ya, donde los agricultores venden sus productos naturales [...]" (Fuente: encuestado de la zona).

En cuanto a la dimensión sociocultural, los habitantes de la comuna argumentan que la instalación de una industria salmonera en la zona amenazaría la seguridad que caracteriza la comuna, a la vez que podría provocar rupturas en las relaciones socio-afectivas de la comunidad. Además, 
consideran que hay un riesgo significativo en provocar una alteración de las costumbres y la identidad del pueblo.

“Hay una amenaza que en este momento no está siendo valorada aparte de la contaminación es que es la intromisión en [...] la parte social de las comunidades ya, si ustedes logran investigar en algún momento la instalación de la salmonera en Chiloé llego a cambiar la, las costumbres, por ejemplo allá existía una moneda de cambio que es el trueque es decir la minga también donde ellos un día todos trabajábamos para Pedrito y al otro día todos pa' Juanito y esa es la forma de pago ya y eso era ancestralmente en Chiloé [...] y eso en ningún momento esta dimensionado de cuanto fue el cambio o el prejuicio que creo". (Fuente: encuestado de la zona).

Una descripción detallada de la información que soporta el análisis de las principales motivaciones individuales, es presentada Cuadro $\mathrm{N}^{\circ} \mathrm{A} 1$, anexo $\mathrm{A}$.

\section{Motivaciones colectivas a través de grupos focales}

En cuanto a la dimensión ambiental, el análisis de los grupos focales ratifica las motivaciones declaradas en las entrevistas, en relación a que la posible instalación de esta empresa traería consecuencias irreparables al ecosistema marino. Se ratifica también el alto grado de conocimiento de la comunidad en relación a los efectos ambientales que genera el cultivo de salmones, en términos de acumulación de sedimentos en el fondo marino. Los habitantes de la comuna argumentan también que, la instalación de balsas y jaulas salmoneras contribuiría a la perdida de fauna autóctona de la comuna, generando mortalidad de peces y lobos marinos, amenazando así a la lobería como santuario de la naturaleza en Cobquecura.

“En primer lugar van a empezar a matar el pescado que anda como le llaman ellos salvaje, porque según ellos traen un pescado que es cuidado que está en jaulas, entonces ese es el pescado que ellos van a consumir y los otros pescados que andan por fuera, los lobos, los pajaritos que andan...eso se acaba completamente". "Los lobos se irían a otro lado, ya no quedaría lobería, porque ellos ya están acostumbrados a comer cosas sanas y ellos se empezarian a enfermar y se comenzarían a extinguir, los mismos pescados contaminados los enfermarían" (Fuente: participantes del grupo focal).

Los resultados evidencian también que, gran parte de las motivaciones de la comunidad con relación a la industria fueron influenciadas, de manera directa, por una visita de una delegación de habitantes de la comuna al sur de Chile, particularmente a la Isla de Chiloé, cuya zona ha sufrido varias crisis que se han vinculado a la industria del Salmón. Posterior a este viaje, los delegados dieron a conocer sus experiencias a los habitantes de la comuna, lo que aumentó el sentimiento de rechazo hacia esta industria. La radio local es uno de los medios de comunicación más importantes que se ha utilizado para hacer esta difusión.

En el ámbito económico, la industria acuícola es también rechazada, ya que se considera que las oportunidades que la empresa les puede brindar en términos de empleo son insuficientes. Los participantes perciben que la industria acuícola generará una cantidad insuficiente de puestos de trabajo a una comuna que necesita una gran fuente laboral. Por otro lado, se argumenta que la 
mano de obra necesaria para el trabajo proporcionado por la industria debe ser especializada, y que la comuna no cuenta con este capital humano, por lo que obligaría a la empresa a traer mano de obra desde otras ciudades.

"Es que al final la salmonera es [...] si la instalan le van a dar trabajo a diez personas de aquí y cuantos habitantes somos" (Fuente: participantes del grupo focal).

A pesar de que las fuentes laborales en la comuna son muy escasas, las personas manifiestan que, al realizar diversas actividades, como la recolección de algas, agricultura y pesca, es posible obtener los ingresos necesarios para mantener su calidad de vida. En este escenario, el turismo se ha transformado en una actividad con el potencial de ser una fuente de ingresos constante en la comuna, generando bienestar a una gran parte de la población. En los últimos años, se han generado grandes inversiones en la zona en esta actividad, como, por ejemplo, la construcción de hoteles, cabañas y restaurantes, los que han proporcionado fuentes laborales a la comuna. En este sentido, los habitantes manifiestan que la irrupción de una industria de esta envergadura en la zona provocaría un retroceso en la actividad turística que se ha mantenido de forma ascendente en los últimos años.

“Está surgiendo, está surgiendo la cosa de trabajo, como que se está difundiendo lo que es turismo, pero esto si fuese lo contrario, esto volvería a cero [...] y con la salmonera yo creo que cambiaría, pero para peor, ya no se vendrían las empresas como ya están llegando cabañas y todo eso le va dando un trabajo a alguien" (Fuente: participantes del grupo focal).

Es importante destacar que los conflictos industria-comunidad no son nuevos en la comuna. En el año 2008, Cobquecura y las comunas vecinas se manifestaron en contra de la empresa forestal celulosa Arauco por la instalación de un ducto que vertería residuos al rio Itata, el cual fue instalado pese al fuerte rechazo de la comunidad. Lo anterior ha reforzado la preocupación de los vecinos, ejerciendo una mayor presión para evitar la instalación de la industria acuícola en la zona. Pese a que la comuna terminó rodeada por grandes empresas forestales, afectando negativamente las fuentes de agua y la actividad agrícola, los habitantes perciben que este sector entrega algunos beneficios a los vecinos como extracción de leña y hongos comestibles, los cuáles se comercializan para generar ingresos. Los participantes del grupo focal realizan un paralelo entre la industria forestal y la acuícola, manifestando que a pesar de que ambas industrias generan efectos negativos, la acuicultura sería más dañina.

Finalmente, en el ámbito sociocultural, los resultados evidencian que la comunidad manifiesta un profundo apego por el territorio, destacando tanto sus características como su belleza y tranquilidad, descartando la posibilidad de un reasentamiento voluntario. No obstante, uno de los fenómenos sociales que afecta a la comuna -y que es recurrente en localidades aisladas- es la migración juvenil. La falta de oportunidades de desarrollo, la lejanía de las instituciones de educación, entre otros, ha impulsado a la población joven a migrar a centros urbanos con mayor densidad poblacional y actividad económica. Sin embargo, pese a la importancia de este fenómeno, debido al limitado impacto que generaría la llegada de la industria acuícola en términos económicos, la comunidad no considera que esta industria podría contribuir a aminorar el fenómeno de migración juvenil. 
Otro cambio social que ha experimentado la comuna de Cobquecura los últimos años es la incorporación de la mujer al trabajo, combinando labores del hogar con trabajos de tiempo parcial. Esta situación ha tenido lugar gracias al turismo, que le ha permitido emprender actividades de artesanía, venta de productos gastronómicos e incluso arriendo de sus propias viviendas para alojamiento turístico. La comunidad siente que la industria podría amenazar este avance en participación de la mujer en el mercado laboral.

"Yo arriendo mi casita en el verano po [...] pesitos que caigan po [...] pesitos que caigan digamos bien venidos sean y así eso da pa [...] pa ir renovando pa ir mejorando digamos la [...] casita" (Fuente: participantes del grupo focal).

Para concluir, los habitantes perciben que las actividades generadoras de ingreso que se desempeñan actualmente en la localidad son menos demandantes, en términos de tiempo y exigencia, con relación al ritmo de trabajo que impondría la industria acuícola o una empresa de cualquier otro rubro. Por esta razón, los habitantes de la localidad no están dispuestos a dejar sus hogares para trasladarse a vivir a una ciudad en donde las fuentes de empleo son muy distintas a las que ellos acostumbran. Una descripción detallada de la información que soporta el análisis de las principales motivaciones colectivas, es presentada en Cuadro $\mathrm{N}^{\circ} \mathrm{A} 2$, anexo $\mathrm{A}$.

\section{Discusión}

La acuicultura es una de las actividades económicas de mayor crecimiento e importancia para el desarrollo de las comunidades costeras y la seguridad alimentaria de los países, dentro de un contexto de sobreexplotación y escases de los recursos marinos. En particular, para el caso chileno, la evidencia soporta su aporte a la creación de empleos, bienestar y reducción de la pobreza en aquellas localidades donde ha operado. Sin embargo, la industria acuícola, y particularmente la del salmón, ha sido cuestionada por sus potenciales efectos adversos sobre el ecosistema marino y los impactos socioculturales que provoca la irrupción abrupta de una industria intensiva en capital, en una comunidad acostumbrada al desarrollo de actividades productivas de menor escala, dentro de relaciones laborales mucho más horizontales y de colaboración. Las áreas más productivas para la expansión de la actividad se encuentran en zonas que habitan comunidades fuertemente arraigadas en estos territorios. Es así como la expansión de la industria acuícola enfrenta el desafío de proponer proyectos sustentables que estén en concordancia con los ejes de desarrollo estratégicos de los territorios. Dentro de este contexto, este trabajo persiguió explorar las motivaciones de los habitantes de una comunidad para rechazar una potencial instalación de un proyecto de la industria acuícola en sus costas. Los resultados sugieren que la incertidumbre con respecto a los problemas que generaría la instalación de una empresa acuícola en la comuna en estudio ha dado lugar a una sociedad del riesgo, lo que va en línea con el enfoque propuesto por Beck (2000). Tanto el análisis de motivaciones individuales como el de motivaciones colectivas evidencia que el riesgo inminente que implica este cambio podría evitarse o minimizase a través de la acción colectiva. Debido a que un conjunto específico de motivaciones aparece en forma recurrente en el análisis, independientemente del enfoque utilizado, se tiene evidencia de la robustez de los resultados. Asimismo, el análisis por dimensiones propuesto da cuenta de la naturaleza y magnitud de este problema, lo que ratifica 
la importancia de incorporar en el análisis sus distintas aristas. Las externalidades negativas que produciría la llegada de esta industria en los ecosistemas marinos y zonas protegidas en la dimensión ambiental van en línea con la evidencia proporcionada por los efectos ambientales de la industria acuícola, en particular la salmonicultura (e.g., Buschmann et al., 2006; Soto \& Norambuena, 2004; Barton \& Murray, 2009; Niklitschek et al., 2013; Sepúlveda et al., 2013). Asimismo, los resultados de la dimensión socio-económica van en línea con ICES (2011), que sugiere que, si bien la llegada de esta industria podría generar beneficios económicos a través de la generación de empleo, el requerimiento de mano de obra altamente especializada dificulta la generación de un pacto real en las comunidades locales. Finalmente, las motivaciones que surgieron en el análisis de la dimensión socio-cultural dan cuenta de los procesos que generan la mayor fuente de incertidumbre en esta sociedad, abarcando desde la forma de asignación del tiempo y la seguridad, hasta el asentamiento de otros individuos que podrían irrumpir el orden social. Todos estos aspectos van en línea con Claude et al. (2000) y Barrett et al. (2002), quienes sugieren que incertidumbres de este tipo generan una disrupción cultural en la sociedad, incentivando a los individuos a hacer frente a dichas amenazas.

La unánime postura al rechazo de la industria acuícola argumentada sobre la base del riego de daño ambiental y social, conllevan a pensar que los habitantes del territorio conciben un modelo de desarrollo que podrían estar más en consonancia con el concepto del buen vivir (sumak kawsay en Kichwa, o suma qamaña en Aymara). Este concepto nace de las aspiraciones de los pueblos originarios de América Latina de Bolivia y Perú, y se extiende posteriormente al debate político y académico en otros sistemas socioeconómicos de América Latina (Gudynas, 2011; Hidalgo-Capitán, 2011; Coraggio, 2013; Vega, 2014; Acosta, 2015). El concepto del buen vivir se revela como opuesto al vivir mejor, promoviendo la búsqueda del tránsito desde el logro de la eficiencia hacia la suficiencia, y desde el antropocentrismo hacia un enfoque biocéntrico, como principio orientador del comportamiento humano (Rodriguez, 2014). Este paradigma está fundamentado en tres elementos centrales: la identidad, la equidad y la sostenibilidad (Cubillo-Guevara et al., 2016). Para el caso de Cobquecura, las opiniones de sus habitantes infieren una preferencia por un modelo de progreso basado en nuevos patrones económicos y que releva la armonía personal, social y con el medio ambiente. En esta última dimensión, las preferencias de la comunidad a promover la economía local a través de actividades de pequeña escala y turismo sustentable podrían estar en línea con la conformación de una sociedad que vive en interacción con la naturaleza, ejerciendo su aprovechamiento y disposición de lo necesario y suficiente dentro de una forma de vida austera (Cabrales, 2015). Esta propuesta, que se postula como una alternativa al discurso neoliberal, capitalista y colonialista, viene a romper el paradigma sobre la visión occidental del desarrollo impuesta a nivel mundial (Cubillo-Guevara et al., 2014; Torres-Solís \& Ramírez-Valverde, 2019). Bajo esta lógica, la comunicación del concepto del desarrollo en el marco del buen vivir requiere un vínculo mucho más estrecho con las comunidades, así como el establecimiento de estrategias participativas (Barranquero-Carretero \& Sáez-Baeza, 2015). Este diálogo ha estado ausente en la relación comunidad-empresa en el marco del proyecto. Es importante destacar que, más allá de visiones fundamentalistas e ideológicas, el concepto de buen vivir puede ser entendido como una propuesta particular, gestada desde América Latina, del discurso contemporáneo del desarrollo sostenible (Vanhulst \& Beling, 2013a; Vanhulst \& Beling, 2013b). 


\section{Conclusión}

A partir de la información recogida, se concluye que la comunidad de Cobquecura presenta una fuerte postura al rechazo a la instalación de la industria acuícola en la comuna. Uno de los principales argumentos que se presentan, se relaciona con el riesgo de daño ambiental que traería una posible instalación de la industria en la zona, principalmente con un riesgo inminente de destrucción de la lobería declarada santuario de la naturaleza, e ícono característico de la zona. Esta postura de rechazo es reforzada con experiencias negativas de esta industria en otras localidades del país.

En relación a los potenciales beneficios económicos, la percepción es que éstos no son tan claros. Lo anterior se debe a que, la demanda de trabajo especializada que se requiere en este tipo de industrias, no beneficiaría potencialmente a los habitantes de la zona. Por consiguiente, el aumento potencial en los puestos de trabajo, traería un efecto positivo principalmente en otras comunas, con un número reducido de beneficiarios locales. Asimismo, los habitantes de Cobquecura perciben que el territorio posee una mayor aptitud para el turismo.

En el ámbito sociocultural, se percibe un fuerte arraigo y un sentido de pertenencia con el territorio, compatible con un estilo de vida pausado, y con un sustento suficiente sobre la base de actividades a pequeña escala en agricultura, pesca artesanal y turismo. Se percibe que la instalación de la industria podría poner el riesgo esta forma de vida, creando relaciones laborales de dependencia, y promoviendo la llegada de personas que no compatibilizan con esta forma de vida.

Finalmente, es importante resaltar el alto grado de organización e involucramiento que ha tenido la comunidad de Cobquecura para oponerse a este proyecto. Una de las razones que declaran los entrevistados y los participantes de los grupos focales para este generalizado rechazado, ha sido el bajo nivel de información y vinculación de la empresa responsable con la comunidad, lo que ha creado un sentimiento de desconfianza hacia ésta, que dificulta el diálogo y negociación para llegar a una resolución del conflicto.

\section{Referencias}

ACOSTA, A. El Buen Vivir como alternativa al desarrollo. Algunas reflexiones económicas y no tan económicas. Política y sociedad, 2015, Vol. 52, № 4, p. 299-330. https://doi.org/10.5209/rev_ POSO.2015.v52.n2.45203

ADAM, B, BECK, U., \& VAN LOON, J. The risk society and beyond. California: Editorial Sage publications Ltd, 2000.

ALFARO, D. \& PEÑA-CORTÉS, F. Potencial acuícola en áreas pre-andinas de la región de la Araucanía: conflictos de uso con la actividad turística. Revista de Geografía Norte Grande. 2012, Vol 51, p. 137-157. http://dx.doi.org/10.4067/S0718-34022012000100008

ALLEN, L., LONG, P., PERDUE, R., and KIESELBACH, S. The impact of tourism development on residents' perceptions of community life. Journal of Travel Research, 1988, Vol. 27, No 1, p. 16-21. https://doi.org/10.1177/004728758802700104 
AMTMANN, C., \& BLANCO, G. Efectos de la salmonicultura en las economías campesinas de la región de Los Lagos, Chile. Revista Austral de Ciencias Sociales. 2017, Vol. 5, p. 93-106. https://doi. org/10.4206/rev.austral.cienc.soc.2001.n5-09

BARRANQUERO-CARRETERO, A., SÁEZ-BAEZA. Ch. La crítica descolonial y ecológica a la comunicación para el desarrollo y el cambio social, Palabra Clave, 2015, Vol. 18, №1, p. 41-82. https:// dx.doi.org/10.5294/pacla.2015.18.1.3

BARRET, G., CANIGGIA, M., \& Read, L. There are more vets than doctors in Chiloe: Social and community impact of the globalization of aquaculture in Chile. World Development. 2002. Vol 30, p.1951-1965. https://doi.org/10.1016/S0305-750X(02)00112-2

BARTON, J.R. \& MURRAY, W.E. Grounding geographies of economic globalisation: Globalised spaces in Chile's non-traditional export sector, 1980-2005. Tijdschrift Voor Economische en Sociale Geografie. 2009, Vol. 100, p. 81-100. https://doi.org/10.1111/j.1467-9663.2009.00503.x

BECK, U. Entorno a la teoría de la sociedad del riesgo. Boletín de la A.G.E.N. 2000, Vol. 30, p. 9-20.

BUSCHMANN, A., \& FORTT, A. Efectos ambientales de la acuicultura intensiva y alternativas para un desarrollo sustentable. Revista Ambiente y Desarrollo. 2005, Vol 3, N²1, p. 58-64.

BUSCHMANN, A. Impacto ambiental de la acuicultura. El estado de la investigación en Chile y el mundo. Osorno: Editorial Terram Publicaciones. 2011.

BUSCHMANN, A., RIQUELME, V., HERNÁNDEZ-GONZÁLEZ, M., VARELA, D, JIMENEZ, J., HENRIQUEZ, L. A review of the impacts of salmonid farming on marine coastal ecosystems in the southeast Pacific. 2006. Ices Journal of Marine Science, Vol. 63, p.1338-1345. https://doi.org/10.1016/j. icesjms.2006.04.021

CABELlO, F., GODFReY, H., TOMOVA, A., IVANOVA, L., DOLZ, H, \& MILLANAO, A. Antimicrobial use in aquaculture re-examined: its relevance to antimicrobial resistance and to animal and human health. 2013. Environmental Microbiology, Vol. 15, p. 1917-1942. https://doi.org/10.1111/14622920.12134

CABRALES, O. El principio del buen vivir o "sumak kawsay", como fundamento para el decrecimiento económico. Cuadernos de filosofía latinoamericana, 2015, Vol. 36, № 113, p. 83-99. https:// doi.org/10.15332/s0120-8462.2015.0113.04

CAMBELL, I., MACLEOD, A., SAHLMANN, C., NEVES, L., FUNDERUD, J., ØVERLAND, M., HUGHES, A., and STANLEY, M. The environmental risks associated with the development of seaweed farming in Europe - Prioritizing key knowledge gaps. Frontiers in Marine Science, 2019, Vol. 22, No 6, p. 1-22. https://doi.org/10.3389/fmars.2019.00107

CEBALLOS, A., DRESDNER-CID, J. \& QUIROGA-SUAZO, M. Does the location of salmon farms contribute to the reduction of poverty in remote coastal areas? An impact assessment using a Chilean case study. 2018. Food Policy, Vol. 75, p. 68-79. https://doi.org/10.1016/j.foodpol.2018.01.009 
CERNEA, M. Reasentamiento de población y estudios sociales. Washington DC: Editorial Banco Mundial, 2001.

CHÁVEZ, C., DRESDNER, J., FIGUEROA, Y. \& QUIROGA, M. Main issues and challenges for sustainable development ofsalmon farming in Chile: a socio-economic perspective. Reviews in Aquaculture, 2019, Vol. 11, N² 2, p. 1-19. https://doi.org/10.1111/raq.12338

CHILVERS, J. Environmental risk, uncertainty, and participation: Mapping an emergent epistemic community. Environment and Planning A: Economy and Space, 2009, Vol. 40; № 12, p. 2990-3008. https://doi.org/10.1068/a39279

CLAUDE, M, OPORTO, J., IBAÑEZ, C., BRIEVA, L., ESPINOZA, C., \& ARQUEROS, M. La ineficiencia de la salmonicultura en Chile: Aspectos sociales, económicos y ambientales. Santiago de Chile: Editorial Terram, 2000.

COACH, S., \& KROLL-SMITH, S. Environmental controversies, interactional resources, and rural communities: siting versus exposure disputes. Rural Sociology, 1994, Vol. 59, No 1, p. 25-44. https://doi.org/10.1111/j.1549-0831.1994.tb00520.x

CORRAGIO, J. L. La economía social y solidaria: hacia la búsqueda de posibles convergencias con el vivir bien. En: FARAH, I. y TEJERINA, V. (eds.). Vivir bien: infancia, género y economía. La Paz: Cides-UMSA, 2015, p. 215-256.

CUBILlo-gUeVARA, A. P., HIDALGO CAPITÁN, A. L., \& DOMÍnGUEZ-GÓMEZ, J. A. El pensamiento sobre el Buen Vivir. Entre el indigenismo, el socialismo y el posdesarrollismo. Revista del CLAD Reforma y Democracia, 2014, Vol. 60, p. 27-58.

CUBILLO-GUEVARA, A.P., HIDALGO-CAPITÁN, A.L., GARCÍA-ÁLVAREZ, S. El Buen Vivir como alternativa al desarrollo para América Latina. Iberoamerican Journal of Development Studies, 2016, Vol. 5, $N^{\circ} 2$, p. 30-57. https://doi.org/10.26754/ojs_ried/ijds.184

CUENCA, L. Celulosa Arauco en Valdivia: El desastre ambiental en el Río Cruces, resultado del modelo forestal chileno. Observatorio Latinoamericano de Conflictos Ambientales, 2005. (Consulta: 12/05/2019). http://olca.cl/articulo/nota.php?id=102067

BECK, U \& REY, J. La sociedad del riesgo global. Madrid: Siglo Veintiuno, 2002.

EZZATI, M., UTZINGER, J., CAIRNCROSS, S., COHEN, A, \& SINGER, B. Environmental risks in the developing world: exposure indicators for evaluating interventions, programmes, and policies. Journal Epidemiol Community Health, 2005, Vol. 59, p. 15-22. http://dx.doi.org/10.1136/jech.2003.019471

FAILING, L., GREGORY, L., and HARSTONE, M. Integrating science and local knowledge in environmental risk management: A decision-focused approach. Ecological Economics, 2007, Vol. 64, No 1, p. 47-60. https://doi.org/10.1016/j.ecolecon.2007.03.010 
FAO. El estado mundial de la pesca y la acuicultura. Contribución a la seguridad alimentaria y la nutrición para todos. Roma: Food and Agriculture Organization of the United Nations, 2016.

FLACSO (Facultad Latinoamericana de Ciencias Sociales). Diagnóstico con Información Secundaria para el Desarrollo Territorial de la Nueva Región de Ñuble. Subsecretaria de Desarrollo Regional del Gobierno de Chile, Santiago, 2018.

GALINDO, J. El concepto de riesgo en las teorías de Ulrich Beck y Niklas Luhmann. Revista Acta Sociologica, 2015. Vol. 67, p.141-164. https://doi.org/10.1016/j.acso.2015.03.005

GELLERT, P. and LYINCH, B. Mega-projects as displacements. International Social Science Journal, 2004, Vol. 55, No 175, p. 15-25. https://doi.org/10.1111/1468-2451.5501002

GIDDENS, A, BAUMAN, Z., LUHMANN, N. \& BECK, U. Las consecuencias perversas de la modernidad. Barcelona: Editorial Anthropos. 1996.

GONZÁLEZ, P. Documento de Introducción Mesa 2 (Inmigrantes e Indígenas. El "otro" en el Conocimiento en Ciencias Sociales). Primer Coloquio de Investigación Social Contemporánea Enero 2009. Facultad de Ciencias Sociales, Universidad de Concepción, Chile. 2009.

GOODMAN, L.A. Snowball sampling. Annals of Mathematical Statistics, 1961, Vol. 32, No 1, p. 148170. https://doi.org/10.1214/aoms/1177705148

GOULDSON, A. Risk, regulation and the right to know: exploring the impacts of access to information on the governance of environmental risk. Sustainable Development, 2004, Vol. 12, № 3, p. 136-149. https://doi.org/10.1002/sd.237

GUDYNAS, E. Buen vivir: Germinando alternativas al desarrollo. América Latina en movimiento, 2011, Vol. 462, p. 1-20.

HIDALGO CAPITÁN, A. L. Economía política del desarrollo. La construcción retrospectiva de una especialidad académica. Revista de economía mundial, 2011, Vol. 28, p. 279-320.

ICES. Report of the Study Group on Socio-Economic Dimensions of Aquaculture (SGSA). Steering group on human interactions on ecosystems. Bremen, 2011.

IRELAND, P, \& THOMALIA, F. The role of collective action in enhancing communities' adaptive capacity to environmental risk: an exploration of two case studies from Asia. PLOS Currents Disasters. 2011, Vol. 3, p.1-16. https://doi.org/10.1371/currents.RRN1279

JAIMOVICH, D., GONZÁLEZ, B., CALBUCURA, J., DEL VALLE, N., \& MALDONADO, C. Conflictos sociales y ambientales en Chile. Iberoamericana, 2014, Vol. 18, № 67, p. 205-230. https://doi. org/10.18441/ibam.18.2018.67.205-230 
MILLANAO, A., BARRIENTOS, M., GÓMEZ, C., TOMOVA, A., BUSCHMANN, A., DOLZ, H., \& CABELLO, F. Uso inadecuado y excesivo de antibióticos: Salud pública y salmonicultura en Chile. Revista Médica de Chile, 2011, Vol. 139, p. 107-118. http://dx.doi.org/10.4067/S0034-98872011000100015

MIRANDA, C. \& ROJAS, R. Occurrence of florfenicol resistance in bacteria associated with two Chilean salmon farms with different history of antibacterial usage. Aquaculture, 2007, Vol. 266, №1-4, p. 39-46. https://doi.org/10.1016/j.aquaculture.2007.02.007

MUNICIPALIDAD DE COBQUECURA. Cobquecura, 2018 (Consulta: 14/06/2019). http://www.cobquecura.cl/.

NIKLITSCHEK, E., SOTO, D., LAFTON, A., MOLINET, C. \& TOLETO, P. Southward expansion of the Chilean salmon industry in the Patagonian Fjords: main environmental challenges. Reviews in Aquaculture, 2013, Vol. 5, p. 172-195. https://doi.org/10.1111/raq.12012

OLSEN, M., \& OSMUNDSEN, T. Media framing of aquaculture. Marine Policy, 2019, Vol. 76, p. 19-27. https://doi.org/10.1016/j.marpol.2016.11.013

PELLIECER, I., VIVAS-ELIAS, P., \& ROJAS, J. La observación participante y la deriva: dos técnicas móviles para el análisis de la ciudad contemporánea. El caso de Barcelona. EURE, 2013, Vol. 39, No 116, p.119-139. http://dx.doi.org/10.4067/S0250-71612013000100005

RINCÓN, M. Territorial conflicts and road infraestructure projects. Bitacora, 2016, Vol. 26, №2, p. 71-78.

RODRÍGUEZ, E. C. Hacia una ética del vivir bien-buen vivir. Producción + Limpia, Vol. 9, N² 2, 2014, p. 11-23. https://doi.org/10.22507/pml

ROJAS, A., SABATINI, F., \& SEPULVEDA, C. Conflictos ambientales en Chile: aprendizajes y desafíos. Revista Ambiente y Desarrollo de CIPMA, Vol. 19, N², p. 22-30.

SEPÚLVEDA, M, ARISMENDI, I, SOTO, D., JARA, F. \& FARIAS, F. Escaped farmed salmon and trout in Chile: incidence, impacts, and the need for an ecosystem view. Aquaculture Environment Interaction, Vol. 4, p. 273-283. https://doi.org/10.3354/aei00089

SOTO, D. \& NORAMBUENA, F. Evaluation of salmon farming effects on marine systems in the inner seas of southern Chile: a large-scale mensurative experiment. Journal of Applied Ichthyology, 2004. Vol. 20, p. 493-501. https://doi.org/10.1111/j.1439-0426.2004.00602.x

STAKE, R. Investigación con estudio de casos. Madrid: Editorial Ediciones Morata, S. L, 1999.

URRA, E., NUÑEZ, R., RETAMAL, C., \& JURE, L. Enfoques de estudio de casos en la investigación de enfermería. Ciencia y Enfermería, 2014, Vol. 1, p. 131-142.

VANHULST, J. \& BELING, A. E. Buen vivir: la irrupción de América Latina en el campo gravitacional del desarrollo sostenible. Revibec: revista iberoamericana de economía ecológica 2013a, Vol. 21, p. 1-14. 
VANHULST, J. \& BELING, A. E. (2013b). El Buen vivir: una utopía latinoamericana en el campo discursivo global de la sustentabilidad. Polis, 2013b, Vol. 12, No 36, p. 497-522. http://dx.doi. org/10.32735/S0718-6568/2013-N36-998

VARGAS, J. Cooperación y conflicto entre empresas, comunidades, nuevos movimientos sociales y el papel del gobierno. El caso de Cerro de San Pedro. Revista de Historia Regional y Local, 2009. Vol. 1, N², p. 86-135.

VEGA, F. El buen vivir-Sumak Kawsay en la Constitución y en el PNBV 2013-2017 del Ecuador. OBETS: Revista de Ciencias Sociales, 2014, Vol. 9, No 1, p. 167-194. http://dx.doi.org/10.14198/ OBETS2014.9.1.06

VILLALOBOS, F. Emol, 2018. (Consulta: 25/10/2019). https://www.emol.com/noticias/Economia/2016/03/01/790812/proyecto-acuicola-en-cobquecura.html. 2016.

WAKEFIELD, S., ELLIOTT, S., COLE, D., and EYLES, J. Environmental risk and (re)action: air quality, health, and civic involvement in an urban industrial neighbourhood. Health and Place, 2001, Vol. 7, No 3, p. 163-177. https://doi.org/10.1016/S1353-8292(01)00006-5

YENNETI, K., DAY, R., and GOLUBCHIKOV, O. Spatial justice and the land politics of renewables: Dispossessing vulnerable communities through solar energy mega-projects. Geoforum, 2016, Vol. 76, p. 90-99. https://doi.org/10.1016/j.geoforum.2016.09.004 


\section{Anexo. \\ Descripción de motivaciones individuales y colectivas por dimensión}

Cuadro $N^{\circ} \mathrm{A} 1$.

Análisis de motivaciones individuales

\begin{tabular}{|c|c|}
\hline DIMENSIÓN & TESTIMONIO \\
\hline Ambiental & $\begin{array}{l}\text {. “.. es una de las loberías más estables del sur del país, centro sur, porque } \\
\text { tiene la particularidad que no todas las otras loberías tienen, no es una lo- } \\
\text { bería estacional es una lobera estabilizada de pariciones, por lo tanto tiene } \\
\text { otro valor sumamente importante en lo que es la conservación de esta...esta } \\
\text { especie". } \\
\text { "Y por otra parte, en aguas superficiales también es invasivo porque están los } \\
\text { caladeros de la pesca artesanal, de la sardina, de la anchoveta, de la merluza, } \\
\text { entonces son los lugares donde los pescadores calan sus redes para hacer } \\
\text { estas capturas y por otro lado también por ahí mismo pasa el tránsito de } \\
\text { ballenas que está siempre rotando a nivel mundial y que por acá se quedan } \\
\text { todos los años bastante tiempo andan con sus crías, tenemos una colonia de } \\
\text { delfines endémicos de acá de la zona...". } \\
\text { "La información que yo sabía es que era un producto bueno...por su color, } \\
\text { sano, pero a medida que me he ido informando y he ido aprendiendo me he } \\
\text { dado cuenta que es un producto toxico ya para la salud humana ya que en } \\
\text { chile se utilizan sobre quinientas veces la cantidad de antibióticos que es } \\
\text { la que se utiliza en noruega donde es el primer productor de salmón ya, sin } \\
\text { contar el deterioro al fondo marino...". }\end{array}$ \\
\hline Económica & $\begin{array}{l}\text { "No nada, porque en primer lugar ellos necesitan una mano de obra especia- } \\
\text { lizada, en segundo lugar, ellos se tienen que embarcar en Talcahuano así que } \\
\text { la gente que va a trabajar va a embarcarse allá y nosotros acá los pescadores } \\
\text { artesanales que somos, de los cuales yo soy su presidenta ninguna le vamos } \\
\text { a trabajar a esa empresa". }\end{array}$ \\
\hline Sociocultural & $\begin{array}{l}\text {. "Llega gente con...muy malas costumbres, muchas veces aparecen delin- } \\
\text { cuentes ya y en la parte social muchas mamas solteras e hijos huérfanos...que } \\
\text { esa parte hasta el momento no ha sido abordada por el proyecto...tenemos } \\
\text { una comunidad sana ya, la gente no es mala, pero eso va... en algún momento } \\
\text { con la intromisión de esta empresa que traen gente de muchos lugares, no } \\
\text { digo que sean todos, pero una manzana echa a perder un cajón, entonces ese } \\
\text { es el problema". }\end{array}$ \\
\hline
\end{tabular}

Fuente: Elaboración propia con base en las entrevistas realizadas a actores clave de la comuna en diversos 
Cuadro $\mathrm{N}^{\circ} \mathrm{A} 2$.

Análisis de motivaciones colectivas

\begin{tabular}{|c|c|}
\hline DIMENSIÓN & TESTIMONIO \\
\hline Ambiental & $\begin{array}{l}\text {. } \text { "No puede tapar uno el sol con un dedo digamos...porque los reportajes para } \\
\text { el sur o las personas que vienen del sur nos dan a entender que nos opon- } \\
\text { gamos rotundamente...que ya para allá tienen su mar muerto...hay agua, hay } \\
\text { mar, pero no hay vida...por las instalaciones que hubo masivas de las indus- } \\
\text { trias esas...no levantaron ni siquiera sus implementos dejaron todo ahí y se } \\
\text { fueron con el dinero en los bolsillos...entonces el mar quedo completamente } \\
\text { muerto...sin seres vivos". } \\
\text {. "A nosotros mismos nos dijeron quizás en un aumento de uno, dos o tres años } \\
\text { no le va a hacer nada, pero cuando empieza ya los residuos a acumularse aba- } \\
\text { jo, eso se esparce y eso es... son las cosas que le inyectan a eso, y el alimento } \\
\text { que se va abajo". } \\
\text { "Fueron los que tienen el sindicato...fueron a Chiloé...porque en Chiloé tienen } \\
\text { una...lo mismo paso...la gente está disconforme...porque han ido a verificar } \\
\text { que si es así po... no porque ellos le contaron, sino que han ido a ver". }\end{array}$ \\
\hline Económica & $\begin{array}{l}\text { "Aparte que los buzos los traen de afuera porque se tiene que meterse a las } \\
\text { jaulas abajo po, y eso son buzos, no son buzos de estos mismos buzos, no le } \\
\text { sirven...porque tienen que ser buzos profesionales". } \\
\text { "Bueno aquí no hay fuentes laborales, pero siempre uno tiene para el día y } \\
\text { algo más también, si eso depende del esfuerzo que le ponga cada uno porque } \\
\text { el flojo siempre le va a faltar, pero a uno no po, aquí todos somos gente de } \\
\text { trabajo" "aquí hay gente que vive de un miserable sueldo, de un sueldo por } \\
\text { ejemplo ya un sueldo mínimo que es ciento veinte lucas, y el resto se lo gana } \\
\text { en el mar o los otros lo ganamos en la agricultura". } \\
\text { "Pero entre el bosque y lo que van a hacer en el mar, es peor lo del mar... } \\
\text { porque del bosque uno saca leña y se cocina o saca callampas y también es } \\
\text { fuente de trabajo para uno...pero en el mar no". }\end{array}$ \\
\hline Sociocultural & $\begin{array}{l}\text { "Nosotros estamos acostumbrados a vivir aquí, es nuestra tierra, estamos ra- } \\
\text { dicadas acá entonces para donde nos van a sacar, aunque vengan a comprar- } \\
\text { nos el terreno y además que es lindo acá. Tenemos que luchar". } \\
\text { "Los que tienen trabajo son los que trabajan en el colegio y demos gracias al } \\
\text { pro empleo que los pagan el sueldo mínimo, trabajamos y si no, no...somos } \\
\text { treinta y dos nosotros los que trabajamos por eso en distintas partes, aquí en } \\
\text { Cobquecura, Taucú, Colmuyado, Buchupureo, Pullay", "Por eso la juventud se } \\
\text { está yendo toda porque aquí no hay en que trabajar", "Los jóvenes que aquí } \\
\text { se quedan en Cobquecura es para que quede perdido nomas". } \\
\text { "Yo le voy a poner un ejemplo la señora que está aquí está acostumbrada a } \\
\text { vender sus mariscales, sabe hacerlos, sabe hacer sus empanadas, sabe hacer } \\
\text { todo lo que es atender a un turista cierto y la van a llevar allá a Chillán que } \\
\text { vaya a vender unas pocas papas, un poco de maíz si ni siquiera sabe cómo se } \\
\text { hace eso, como vamos a ir a meternos a Quirihue, por lo menos cuando aquí } \\
\text { ya están acostumbrados a hacer su pan, venderlo tranquilamente". }\end{array}$ \\
\hline
\end{tabular}

Fuente: Elaboración propia con base en los grupos focales donde participaron miembros de organizaciones sociales 
